\title{
Research on reverberation characteristics analysis and suppression methods for active continuous detection
}

\author{
Tuo Chen", Qiming Ma, Zheyuan Zhou, and He Lu \\ Science and Technology on Sonar Laboratory, Hangzhou Applied Acoustics Research Institute, Hangzhou City, Zhejiang \\ Province, China
}

\begin{abstract}
Compared with the traditional active detection with monopulse periodic emission mode, active continuous detection has the advantages of large emission duty ratio and continuous acquisition of process information. It can effectively overcome the disadvantages of high false alarm probability, poor environment adaptation ability and low detection efficiency in traditional active detection, so then improving the system detection ability. But active continuous detection is also facing more serious reverberation. In order to further improve capacity, the adaptable reverberation characteristics and detection methods are carried out in this paper. On the basis of theoretical modelling, the relationship between the characteristics of the active continuous detection reverberation and the signal form, the hydrological environment and the emission power are studied. The time frequency characteristics of reverberation and the attenuation law with distance of reverberation are mastered. A reverberation suppression method based on adaptive beamforming of sub-band steered minimum variance algorithm (SSMV) is studied for active continuous detection. Considering signal bandwidth and fast convergence, etc. The relationship between sub-array partition and reverberation suppression ability is analyzed. The validity of reverberation characteristic analysis is verified by simulation, the performance of the method of reverberation suppression is verified by sea trial data processing.
\end{abstract}

\section{Introduction}

With the improvement of the distance of the active sonar and the wide application of the remote range, the shortcomings of the tradition active sonar detection system are gradually exposed, that is, the high false alarm rate, the unstable detection performance, and the frequent loss of the target phenomenon. In the research field of active sonar detection technology, the active continuous detection technology is gradually paid attention to. The concept of active continuous detection is first proposed in the field of radar technology and has been developed rapidly. The biggest feature of active continuous detection is to adopt continuous wave signal or multipulse waveform signal transmitting and continuous receiving mode to realize the reception of target echo waveform. At present, the research of active continuous detection technology mainly focuses on signal waveform design, echo signal/information processing and so on. Hickman and L. Krolik proposed a form of multi-carrier frequency linear frequency modulation (LFM) signal whose carrier frequency was determined by Costas sequence. The disadvantage of the waveform was that the center frequency interval must be guaranteed[1]. De Ferrari and Wylie proposed the M sequence coded pulse signal, which has a good distance to radial velocity resolution, but its disadvantage is that it

* Corresponding author: $\underline{\text { skl chent@163.com }}$ is easily affected by the spatio-temporal correlation of the ocean channel, which leads to the difficulty of ensuring the phase information that its matched filtering depends on[2]. Simon J.L through different combinations of frequency hopping signals, it achieves the separation of direct or reverberation signals from echo signals[3]. In the related literature, Doug. G discussed the Doppler estimation and target tracking based on the continuous $\mathrm{CW}$ signal active continuous detection, and pointed out that the target distance cannot be accurately estimated for single generation and single collection configuration, but the target distance and speed can be realized simultaneously by data fusion and target tracking for multiple-input and multiple-output system configuration[4].

The factors affecting the performance of active continuous detection include ship noise, marine environment noise and reverberation is the main factor. The reverberation comes from the acoustic scattering caused by the inhomogeneity of the sea water and the scattering of the sea surface and the seabed. It is a comprehensive result of propagation and scattering, which is related to both the propagation environment and the various scatterers[5]. For the moment, the research on reverberation suppression in active sonar signal processing mainly focuses on spatio-temporal and timefrequency domain processing[6].In this paper, based on 
the reverberation theory modelling, the characteristics of active continuous detection sonar are studied. The application of the SSMV adaptive beamforming algorithm in the active continuous detection of reverberation suppression is carried out from the wideband space-time characteristics by using the coherent cumulative features[7,8,9]. Based on the above research, the effectiveness and performance of the proposed method are verified by numerical simulation and real data processing.

\section{Analysis of reverberation characteris- tics of active continuous detection}

\subsection{Active continuous detection reverberation model}

Under shallow water environment, seafloor reverberation is the most important factor affecting active detection[10]. The theory of underwater reverberation point scattering model is used for reference in this paper. Assuming that scatterers are randomly distributed over the entire seabed, the modelling of reverberation characteristics for active continuous detection is carried out in combination with the characteristics of transmitted signals from active continuous detection. To simplify the calculation, make the following assumptions:

- Suppose the sound wave propagates linearly, only the attenuation of spherical wave is considered, and the multipath effect is not considered.

- Any instantaneous scatterer is randomly distributed evenly in any region of the seabed.

- The angular variation between the scatterer and the receiver caused by the active sonar platform is neglected in a sufficiently short analysis time, we also ignore the propagation effect in the scale of area element.

Fig. 1 shows a schematic diagram of the seabed point scattering model.

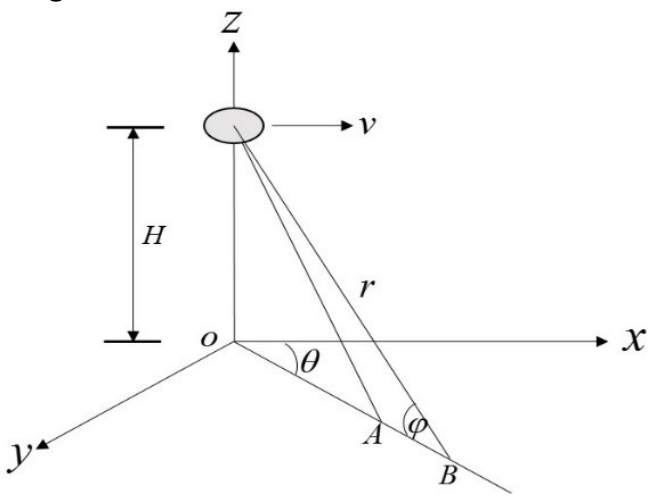

Fig. 1. Seabed point scattering model.

The most prominent feature of active continuous detection is long pulse width or pulse string signal. The corresponding reverberation time series is the accumulation of the scattered signal echoes in the scattering area affected by the transmitted signal. From a statistical point of view, the instantaneous value of reverberation satisfies the Gaussian distribution, the envelope satisfies the Rayleigh distribution, and the instantaneous phase satisfies the uniform distribution. In this paper, the active sonar system under the condition of both receiver and transmitter, the reverberation can be expressed in the follow form in engineering

$$
y(t)=\sum_{n=1}^{N} A_{n} s\left[\eta_{n}\left(t-\tau_{n}\right)\right]
$$

The incidence azimuth of the emitter $\theta \in\left(-90^{\circ}, 90^{\circ}\right)$, the height of platform to seafloor is $H$, the motion velocity is $v, N$ denotes the number of the scatter, $A_{n}$ denotes the amplitude of the nth revolution $s(\ldots)$ denotes scattering echo, $\eta_{n}$ denotes the Doppler scale factor of the $n$th scatterer, $\tau_{n}$ denotes time delay of the nth scattering echo received by the receiver.

Such as shown in Fig.1, the distance A and B to platform is $r_{\min }$ and $r_{\max }$, the pitching angle is $\varphi, N$ scatterers are distributed at a very small distance $d r$, these scatterers are distributed in the semicircle of the seabed, the total area of the scatterers is

$$
S_{a}=\pi \sqrt{r^{2}-H^{2}} d r / \cos \varphi
$$

So the area represented by a single scatterer A with a distance of $r$ is

$$
S_{n}=\frac{\pi r}{N}\left(r_{\max }-r_{\min }\right)
$$

The influence of absorption and attenuation of seawater and the directivity of emission and reception is neglected. The scattering intensity is obtained by Lambert's law

$$
S_{b}=10 \log _{10} u+10 \log _{10}\left(\sin ^{2} \varphi\right)
$$

The proportionality constant is $u=10^{-2.7}$, the amplitude of echo is

$$
A_{n}=10^{\left(S_{b} / 10\right) S_{n}}
$$

Doppler factor of echo is

$$
\eta_{n}=\left(c-v_{n} \cos \theta_{n} \cos \varphi_{n}\right) /\left(c+v_{n} \cos \theta_{n} \cos \varphi_{n}\right)
$$

As shown above, $c$ is sound velocity of sea water, the time delay of echo is

$$
\tau_{n}=2 r_{n} / c
$$

The reverberation bandwidth will be broadened compared with the transmitted signal. On one hand, the scatterers in the ocean are moving in different directions, resulting in Doppler effect and random phase shift, on the other hand, it is related to the time and shape of the transmitted signal. If the transmitted signal is LFM, the reverberation bandwidth roughly is:

$$
R_{B}=B_{L F M}+2 / T
$$

In (8), $R_{B}$ denotes the bandwidth of reverberation, $B_{L F M}$ is the bandwidth of the transmitted LFM signal, $T$ denotes pulse width of the transmitted LFM signal. 


\subsection{Reverberation characteristic simulation and analysis for active continuous detection}

Based on the model established in section 2.1, the reverberation characteristic is analyzed through the simulation method. The relationships between reverberation bandwidth, frequency spectrum, the attenuation rule and the transmitted signal pulse width, hydrologic condition, seabed properties are special analyzed. Condition of the simulations are listed such as, transmitted signal is LFM. Centre frequency is $1550 \mathrm{~Hz}$. spectrum bandwidth is $100 \mathrm{~Hz}$. Pulse width is selected $4 \mathrm{~s}$ and $64 \mathrm{~s}$ separately for the convenience of comparison. Sampling frequency is $30 \mathrm{kHz}$. Sound velocity is $1500 \mathrm{~m} / \mathrm{s}$. Active sonar type is both receiving and transmitting. The sea depth is $200 \mathrm{~m}$, transmitting and receiving depth is $50 \mathrm{~m}$. The source level of transmitting is $220 \mathrm{~dB}$.

\subsubsection{Time characteristic of reverberation}

Firstly, the time characteristic of reverberation is analyzed for different pulse width signal. Results are shown in Fig.2.

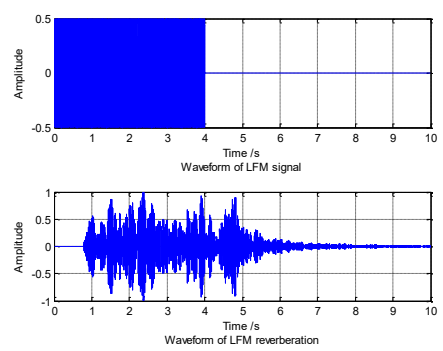

(a) 4 s pulse width
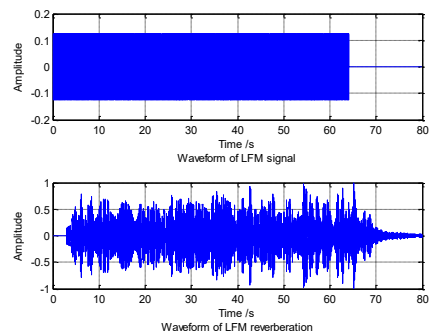

(b) $64 \mathrm{~s}$ pulse width

Fig. 2. Transmitting signal and corresponding reverberation time wave form.

From Fig.2, we can see that the influence range of reverberation is enlarged proportional to the pulse width of the transmitting signal. For active continuous detection, the signal is transmitted continual; the target detection will be effected by the reverberation in all time. In order to enhance the performance of the active continuous detection, some reverberation suppression method must be developed.

\subsubsection{Frequency characteristic of reverberation}

Secondly, the frequency characteristic of reverbera-tion is analyzed for different pulse width signal. Results are shown in Fig.3.
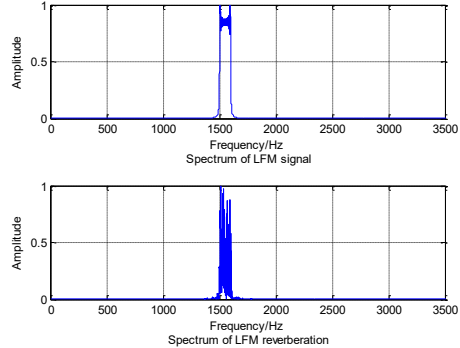

(a) 4 s pulse width
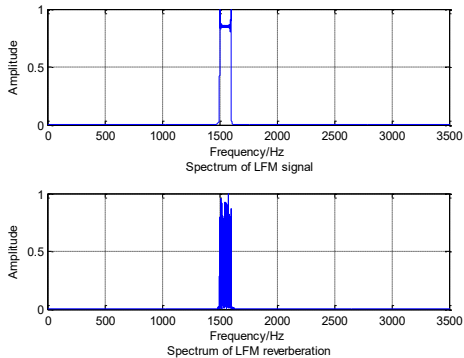

(b) $64 \mathrm{~s}$ pulse width

Fig. 3.Transmitting signal and corresponding reverberation spectrum.

From Fig.3, we can see that regardless of the width of the transmitted signal, the reverberation spectrum formed by the superposition of the signal is shown to be broadened in frequency band. At the same time, there are some burrs in the reverberation spectrum, that is, frequency distortion occurs, indicating that the correlation between reverberation and target echo becomes weaker. The broadening of the frequency band will have a certain influence on the subsequent time frequency segmentation research, but this is not the focus in this paper, and the information processing research that matches the active continuous detection will be carried out in the future.

\subsubsection{Attenuation law of reverberation with distance under different sound speed profile (SSP)}

Finally, based on the normal mode, the reverberation attenuation law under different transmitting signal pulse width conditions is analyzed. Results are shown in Fig.4Fig.6.

From Fig.4-Fig.6, we can see that the reverberation attenuation trend is generally the same under different pulse width conditions, but in terms of magnitude, the reverberation strength of $64 \mathrm{~s}$ pulse width signal is greater about $12 \mathrm{~dB}$ than that of $4 \mathrm{~s}$ pulse width signal, and its impact distance also increases. Normally, the spectrum level of marine environment noise at $1 \mathrm{kHz}$ is $64 \mathrm{~dB}$. Supposing the attenuation of the marine environment noise spectrum is $6 \mathrm{~dB} / \mathrm{oct}$, the spectrum level at $1.5 \mathrm{kHz}$ is about $58 \mathrm{~dB}$. Then, under three type hydrology conditions, ie, equal SSP and weakly negative gradient SSP and intensively thermocline SSP, the reverberation influence distance caused by $64 \mathrm{~s}$ pulse width transmitting signal is about $41 \mathrm{~km}, 22 \mathrm{~km}$ and $40 \mathrm{~km}$ respectively. Compared with $4 \mathrm{~s}$ pulse width transmitting signal, the reverberation influence distance is increased about $20 \mathrm{~km}, 10 \mathrm{~km}$ and $17 \mathrm{~km}$. When the pulse width is 
further increased, then the reverberation influence distance will also increase. This will affect the performance of the active continuous detection to a certain extent. Therefore, in practice, the reverberation suppression should be considered.

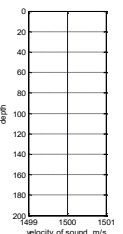

(a) SSP

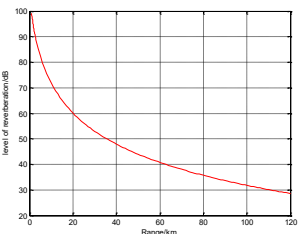

(b) 4 s pulse width

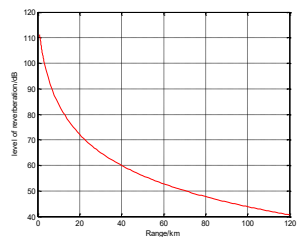

(c) $64 \mathrm{~s}$ pulse width
Fig. 4. Attenuation rule of reverberation with distance under the condition of equal SSP.

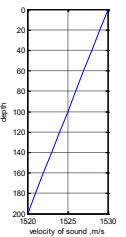

(a) SSP

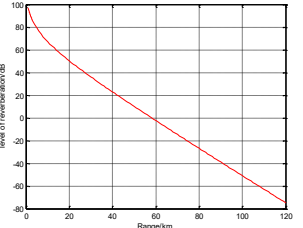

(b) $4 \mathrm{~s}$ pulse width

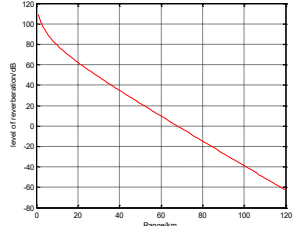

(c) $64 \mathrm{~s}$ pulse width
Fig. 5. Attenuation rule of reverberation with distance under the condition of weakly negative gradient SSP.

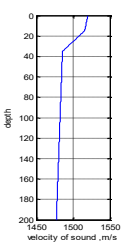

(a)SSP

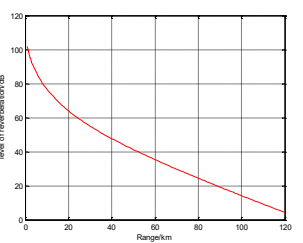

(b) $4 \mathrm{~s}$ pulse width

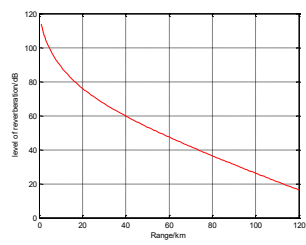

(c) $64 \mathrm{~s}$ pulse width
Fig. 6. Attenuation rule of reverberation with distance under the condition of intensively thermocline SSP.

\section{Reverberation suppression methods for active continuous detection}

From the analysis of reverberation characteristics in section 2, we can see that the reverberation influence is more serious in active continuous detection application, its target detection performance will be effected seriously. Analyzed from many kinds of documents and reports, commonly used reverberation suppression methods are adaptive filtering, high resolution adaptive beamforming, etc. In this section, the basic principles of above methods are introduced, in addition, the existing problems and applicability of these methods when applied to active continuous detection are analyzed specially.

\subsection{Common methods of reverberation suppression}

\subsubsection{Adaptive filtering method}

Adaptive reverberation cancellation uses a reference signal which is strongly correlated with the reverberation signal but weakly correlated with the target echo signal, then the adaptive algorithm is used to dynamically adjust the parameters of the filter, and the reference input signal can be well counteracted with the reverberation component in the received signal after the adaptive filter, so that the output error is the desired target echo signal. However, there are some problems such as inaccurate reference signal and incomplete reverberation suppression, which can not be applied to active continuous detection.

\subsubsection{High resolution adaptive beamforming}

According to the reverberation model, the intensity of the reverberation is proportional to the reflective area of the seabed. Reducing the transmitting or receiving beams is an effective way to reduce the reverberation intensity. The high resolution adaptive beamforming utilizes the difference of the background and the spatiotemporal correlation of the target. The maximum suppression of the noise/interference background is realized on the premise of ensuring the undistorted output of the target beam. At present, commonly used adaptive beamforming methods are minimum varance distortionless response (MVDR), steered minimum variance algorithm (STMV) and sub-band steered minimum variance algorithm (SSMV) etc. Among them, MVDR has slow convergence speed and poor waveform quality in time domain. STMV has many problems such as large computation and coherent cancellation of signal/noise. SSMV can effectively solve the problem of excessive computation by sub-array/band pre-partitioning, but still can not solve the problem of signal/noise coherent cancellation. In addition, the subarray/band division of SSMV is also an important factor determining the performance of its interference suppression. Based on the above analysis, adaptive beamforming such as MVDR and STMV and SSMV can not be completely applied to the problem of reverberation suppression for active continuous detection. For this reason, a reverberation suppression method based on SSMV for active continuous detection is proposed, which is also using forward/backward smoothing method. Through the actual data processing, the relationship between the subarray division and the reverberation suppression performance is analyzed. On this basis, the best subarray division pattern is given for the specific reverberation type.

\subsection{Reverberation suppression method based on SSMV for active continuous detection}

Large aperture array is commonly used in modern sonar. In order to reduce the computational complexity and reduce the dimension of cross-spectral density matrix, the entire array can be divided into several sub-arrays, perform convenntional beamforming for each sub-arary, and make the output of each sub-array beam as the new base element, and carry out STMV adaptive beamforming processing for the new base element, this is the key idea of SSMV. The number of sub-array division is closely related to the performance of reverberation suppression. In practical application, subarray division pattern should be determined by 
considering the ability of reverberation suppression and the processing mode of continuous pulse section.

The predicted frequency domain output of SSMV is:

$$
U(\theta, f)=K_{N} K^{T} X(\theta, f)
$$

In (9), $K_{N}=\operatorname{diag}\left(1 / N_{1}, 1 / N_{2}, \cdots, 1 / N_{J}\right)$ denotes normalization matrix, $X(\theta, f)$ denotes array receiving signal matrix, $N_{j}(\mathrm{j}=1,2, \cdots, \mathrm{J})$ denotes the sub-array element number of the jth sub-array. Each column of the matrix $K$ represents the selected structural sub-array, it is composed of 1 and 0 , indicating whether the element is selected. Then the SSMV covariance matrix and the weight vector are,

$$
\begin{gathered}
R_{S A}(\theta, f)=\sum_{f} U(\theta, f) U^{H}(\theta, f) \\
\boldsymbol{W}_{S A}(\theta)=\frac{\boldsymbol{R}_{S A}^{-1}(\theta, f) \times I_{J}}{I_{J}^{H} \times \boldsymbol{R}_{S A}^{-1}(\theta, f) \times I_{J}}
\end{gathered}
$$

In (11), I is a unit vector of $J \times 1$ vector. Thus, in the $\mathrm{f}$ band, the beam output in the $\theta$ direction can be expressed as

$$
Y_{\text {out }}(\theta, f)=\boldsymbol{W}_{S A}^{\mathrm{H}}(\theta) \boldsymbol{X}_{T}(\theta, f)=\boldsymbol{W}_{S A}^{\mathrm{H}}(\theta) \boldsymbol{T}(\theta, f) \boldsymbol{X}(f)
$$

In (12), $\boldsymbol{T}(\theta, f)$ is the focusing matrix. The Inverse Fast Fourier Transform (IFFT) of $Y_{\text {out }}$ can get the time domain waveform data of the beam.

\subsection{Numerical simulation analysis}

The numerical simulation of the reverberation suppression performance of the SSMV is taken out in this part. Condition of the simulations is listed such as, the number of elements is 64 , and the transmitted signal is LFM. Centre frequency is $1550 \mathrm{~Hz}$. spectrum bandwidth is $100 \mathrm{~Hz}$. The pulse width is $15 \mathrm{~s}$. Sampling frequency is $30 \mathrm{kHz}$. Sound velocity is $1500 \mathrm{~m} / \mathrm{s}$. Active sonar type is both receiving and transmitting. Results are

\begin{tabular}{|c|c|c|c|c|c|}
\hline \multirow{2}{*}{$\begin{array}{c}\text { algorithm } \\
\mathrm{CBF}\end{array}$} & \multirow[t]{2}{*}{$\begin{array}{c}\text { attenuatio } \\
\text { n rate } \\
(\mathrm{dB} / \mathrm{km}) \\
(10.6 \mathrm{~km}, \\
\text { range }) \\
7\end{array}$} & \multicolumn{2}{|c|}{$\begin{array}{c}\text { Reverberation } \\
\text { level }(\mathrm{dB}) \\
\text { (located in } 9.4 \mathrm{~km} \text {, } \\
20 \mathrm{~km})\end{array}$} & \multicolumn{2}{|c|}{$\begin{array}{c}\text { Reverberation } \\
\text { level } \\
\text { difference } \\
\text { between } \\
\text { SSMV and } \\
\text { CBF(dB) } \\
\text { (located in } \\
9.4 \mathrm{~km}, 20 \mathrm{~km}) \\
\end{array}$} \\
\hline & & 65.3 & -8.5 & - & - \\
\hline SSMV-8 & 13.2 & 52.7 & -87.5 & 12.6 & 79 \\
\hline SSMV-4 & 13.5 & 52.7 & -90 & 12.6 & 81.5 \\
\hline SSMV-2 & 14 & 52.7 & -92.6 & 12.6 & 84.1 \\
\hline
\end{tabular}
shown in Fig. 7 and Table 1.

Table 1. Attenuation rate and level of reverberation under different sub-array division conditions.

The relationship between sub-array division and reverberation suppression performance is analyzed and compared. It can be seen that this method has good reverberation suppression performance. Specific numerical analysis can be obtained from Table 1.

The results of Table 1 shows that, for the range of reverberation, the proposed method is effective in the suppression of reverberation under different sub-array conditions, and the maximum difference is more than $80 \mathrm{~dB}$ compared with the CBF. Under each sub-array division condition, the reverberation attenuation rate is about twice as much, and the 2 sub-array conditions can be more than doubled.

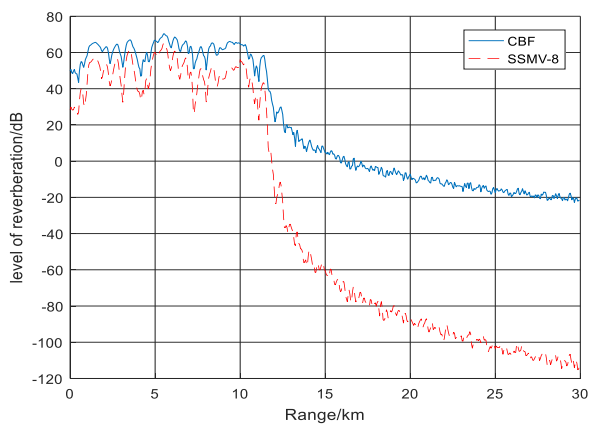

(a) 8 sub-arrays

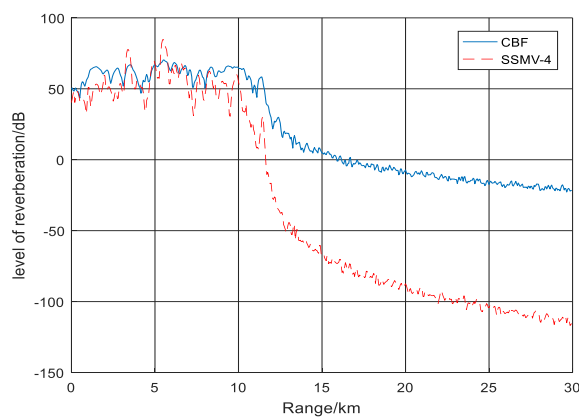

(b) 4 sub-arrays

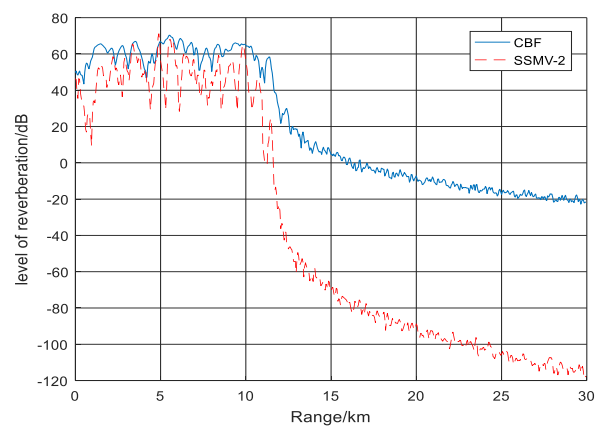

(c) 2 sub-arrays

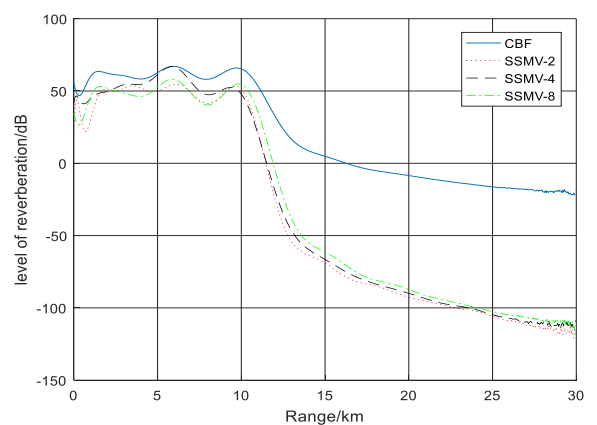

(d) Total

Fig. 7. Reverberation attenuation curve of CBF and SSMV (with different sub-array number ). 


\section{Actual data processing}

In this section, the actual sea trial data are used to analyse the reverberation characteristics, and verify the reverberation suppression performance of the proposed method. In the real sea trial, the transmitting signal is a pulse string signal, the pulse width of a single pulse is 4 seconds, repeat 16 times, the receiving array is a towed line array with 128 channel elements.

\subsection{Comparison of the reverberation characteri- stics of long and short pulse}

The above figures are the result of a sea trial data processing. In Fig.8(a), the attenuation curve of reverberation with the distance under the condition of $4 \mathrm{~s}$ pulse width is shown. In Fig.8(b), the attenuation curve of reverberation with the distance under the condition of $4 \mathrm{~s}$ pulse width repeating 16 times is shown. The reverberation influence distance is $35 \mathrm{~km}$ and $100 \mathrm{~km}$, which shows that the reverberation distance increases with the increase of pulse width or equivalent pulse width. This is consistent with the attenuation law of the previous simulation analysis.

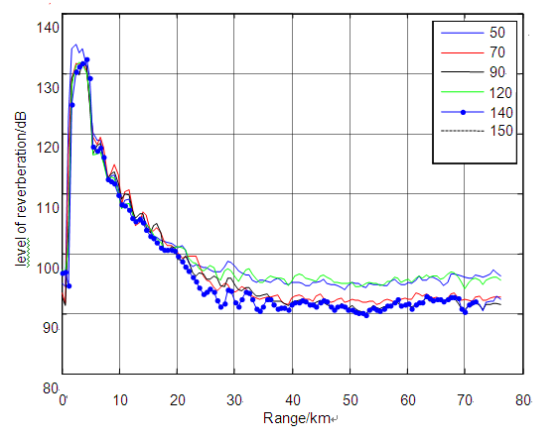

(a) 4 s single

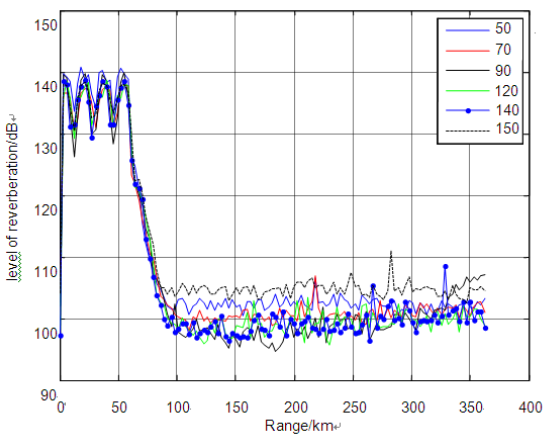

(b) 16 pulse

Fig. 8. Reverberation attenuation curve of $4 \mathrm{~s}$ single pulse and pulse string with 16 pulse.

\subsection{Performance verification of reverberation suppression for proposed method}

In this section, the reverberation suppression performance of the proposed method is verified by the real sea trial data. The relationship between sub-array division and reverberation suppression performance is analyzed and compared, and the number of sub-array partition is determined on the basis of this.Results are shown in Fig. 9 and Table 2.
Table 2. Attenuation rate and level of reverberation under different sub-array division conditions.

\begin{tabular}{|c|c|c|c|c|c|}
\hline \multirow{2}{*}{$\begin{array}{c}\text { algorithm } \\
\mathrm{CBF}\end{array}$} & \multirow{2}{*}{$\begin{array}{c}\begin{array}{c}\text { attenuation } \\
\text { rate }(\mathrm{dB} / \mathrm{km}) \\
(66 \mathrm{~km} \\
\text { range })\end{array} \\
0.62\end{array}$} & \multicolumn{2}{|c|}{$\begin{array}{c}\text { Reverberation } \\
\text { level }(\mathrm{dB}) \\
(\text { located in } \\
64 \mathrm{~km}, 130 \mathrm{~km})\end{array}$} & \multicolumn{2}{|c|}{$\begin{array}{c}\text { Reverberation } \\
\text { level difference } \\
\text { between SSMV } \\
\text { and } \mathrm{CBF}(\mathrm{dB}) \\
(\text { located in } \\
64 \mathrm{~km}, 130 \mathrm{~km})\end{array}$} \\
\hline & & 133.5 & 92.2 & - & - \\
\hline SSMV-16 & 1.14 & 127.6 & 52.3 & 5.9 & 40 \\
\hline SSMV-8 & 1.22 & 127.7 & 47 & 6 & 45.2 \\
\hline SSMV-4 & 1.25 & 127.6 & 45 & 5.9 & 47.2 \\
\hline
\end{tabular}

\section{Conclusions}

The reverberation characteristics of active continuous detection and the application of SSMV in reverberation suppression are studied. Based on the basic concept and classification of the reverberation, the reverberation model for active continuous detection is set up. The relationship between the time characteristics, frequency characteristics, the SSP, the seafloor sediments and the pulse width of the emission signal is simulated and analyzed by the normal mode. The reverberation attenuation law is provided in this paper. Based on the large aperture sonar array, the relationship between the pattern of sub-array division and the reverberation suppression performance is also analyzed. The simulation and actual sea trial data analysis show that the reverberation characteristic analysis method used in this paper is effective. The capacity of the proposed reverberation suppression algorithm exceeds $40 \mathrm{~dB}$ to conventional method, and the reverberation attenuation rate is more than doubled.

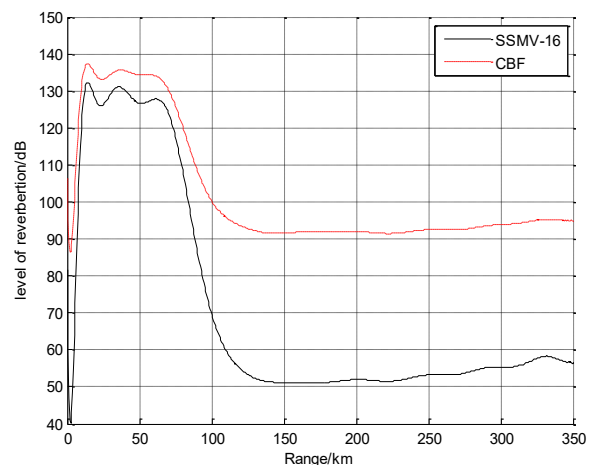

(a)16 sub-arrays

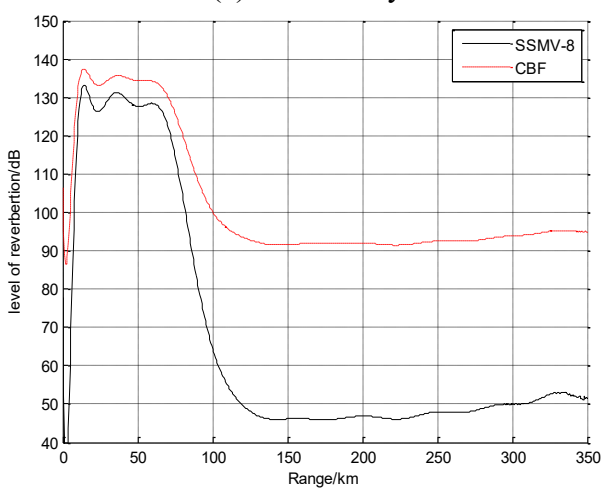

(b) 8 sub-arrays 


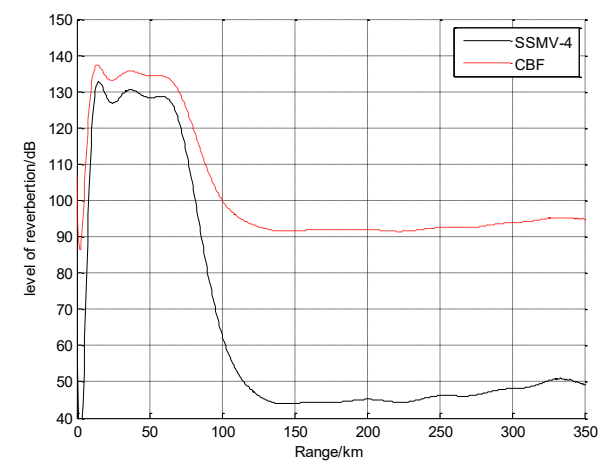

(c) 4 sub-arrays

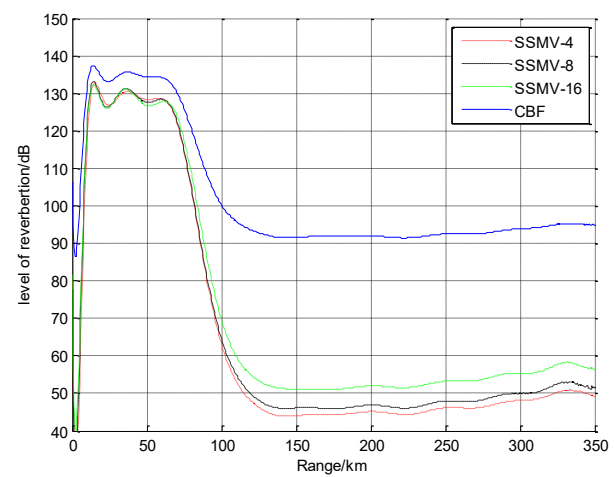

(d) Total

Fig. 9. Reverberation attenuation curve of $\mathrm{CBF}$ and SSMV(with different sub-array number ).

\section{References}

1. Granger Hickman, Jeffrey L. Krolik, Oceans 2012, 1-6(2012)

2. Harry De Ferrari and Jennifer Wylie, International Congress on Acoustics 2013 Montreal. Proceedings of Meetings on Acoustics, Acoustical Society of America. 19(2013)

3. Simon J. Lourey. ICASSP, 1832-1835(2015)

4. Doug Grimmett, Cherry Wakayama, 16th International conference on information fusion Istanbul, Turkey, July9-12, 258-265(2013)

5. Yang Shi'e, Theory of Underwater Sound Propagation. Harbin Engineering University Press, (2009)

6. Zou lina, Tan Ke, Zha Jilin, 2016 IEEE/OES China Ocean Acoustics Symposium.

7. Krikuj J, Swingler D,IEEE Trans. Acoust. Speech Signal Processing, 37(10) 1481-1494(1989)

8. Swingler D, Steered adaptive subarray beamforming DREA-CR-1999-089. Defense R\&D Canada, (1999)

9. Swingler D, IEEE Trans Signal Processing, 47(4)1154-1160(1999)

10. Caruthers J W, Novarini J C, IEEE Journal of Oceanic Engineering, 18(2)100-107(1993) 